\title{
Abnormal Epigenetic Modifications in Surviving Somatic Cell Cloned Cattle Associated With Donor Cell Type
}

Haoqiang Ma

Henan University

Tian Huang

Henan University

Jin Zhou

Henan University

Jinghui Li

Henan University

Shenghui Cui

Henan University

Chongyuan Ye

Henan University

Chao Lu

Henan University

Yueyu Bai

Henan University

Gengsheng Cao ( $\sim$ gscao@henu.edu.cn )

Henan University

\section{Research}

Keywords: somatic cell nuclear transfer, ATAC-seq, RNA-seq, 5hmC, 5mC

Posted Date: September 14th, 2021

DOI: https://doi.org/10.21203/rs.3.rs-853162/v1

License: (c) (i) This work is licensed under a Creative Commons Attribution 4.0 International License.

Read Full License 


\section{Abstract \\ Background}

Studies have shown that the efficiency of somatic cell nuclear transfer (SCNT) is related to the type of donor cell used. Previous studies have shown that fetal oviduct epithelial cells (FOVs) exhibit a higher blastocyst formation rate than fetal fibroblasts (FFBs), but they are associated with lower pregnancy, calving, and full-term rates after implantation. The reason for this difference is unclear.

\section{Result}

In this study, we performed the assay for transposase-accessible chromatin with high-throughput sequencing (ATAC-seq), RNA-seq, and 5-hydroxymethylcytidine (5hmC) and 5-methylcytosine (5mC) DNA methylation sequencing methods across the whole genome to analyze the epigenetic differences between cattle cloned using FFBs or FOVs as donor nuclei. The results showed that chromatin openness, gene expression levels, and $5 \mathrm{hmC}$ contents were greater in cloned cattle derived from FOV donor nuclei than in those derived from FFBs. ATAC-seq and RNA-seq analyses of cloned bovine ear tissues derived from the same source of donor nuclei showed an obvious clustering tendency. In this study, we also found that the $5 \mathrm{hmC}$ content of surviving cloned cattle derived from FFBs was greater than $4 \%$, whereas it was less than $2 \%$ in dead cloned cattle.

\section{Conclusion}

We found that there were abnormalities in specific epigenetic modifications and gene expression in living somatic cell cloned cattle derived from different donor nuclei. Although cloned cattle undergo somatic reprogramming and differentiation, they retain the epigenetic imprints of their donor nuclei, and this somatic imprinting may affect the development rate of cloned blastocysts as well as the birth rate and development status of cloned fetuses after implantation.

\section{Introduction}

Increasing experimental evidence is demonstrating that the problems associated with cloned animals, such as their low birth rates and abnormal postnatal development, are related to abnormal epigenetic modifications in animals derived from somatic cell nuclear transfer reprogramming. Most of the studies conducted to date on epigenetic modifications, including DNA methylation and a variety of histone modifications, have focused on preimplantation embryos. However, the development of cloned animals involves two main stages: the somatic cell nucleus reprogramming stage and the subsequent forward programming developmental stage. Abnormal occurrences in either of these stages will affect the birth rate and developmental status of a cloned animal. 
Most studies on somatic cell nuclear transfer (SCNT) have focused on preimplantation embryos. Compared with in vitro-fertilized embryos, preimplantation nuclear transfer embryos tend to be incompletely reprogrammed. Before active demethylation has been completed, DNA remethylation occurs prematurely in the 4-cell stage, rather than in the 8-cell stage of these embryos [1-3]. Moreover, H3K9me3 modifications of histones are widely enriched in reprogramming-resistant regions. This type of modification affects the early embryo reprogramming efficiency of reconstructed mouse embryos and is the main limiting factor for SCNT reprogramming [4]. Importantly, compared to in vitro-fertilized embryos, the histone acetylation levels of nuclear transfer embryos derived from FFBs are lower, and the reduced histone acetylation content leads to compaction of the chromatin structure, which can be alleviated by the use of the histone deacetylase inhibitor trichostatin A (TSA).

Histone $\mathrm{H} 3 \mathrm{~K} 4 \mathrm{me} 3$ is an epigenetic modification associated with active gene expression. In the reprogramming phase, the $\mathrm{H} 3 \mathrm{~K} 4 \mathrm{me} 3$ modification is beneficial for reprogramming, while in the forward programming stage, $\mathrm{H} 3 \mathrm{~K} 4 \mathrm{me} 3$ may play an important role in donor cell epigenetic memory and cause a failure of genes that are specifically expressed in donor cells to be effectively turned off, resulting in abnormal development [5]. In addition, H3K27me3 is a modification related to gene suppression that plays an important role in maintaining pluripotency in embryonic stem cells. However, H3K27me3 is barely detectable in the inner cell mass of somatic nuclear transfer blastocysts. The abnormal deletion of this modification leads to the premature expression of developmental genes in the forward programming stage and the death of somatic cloned embryos shortly after implantation $[6,7]$.

A number of studies conducted to date have revealed a correlation between accessible chromatin regions and histone modifications in mouse preimplantation embryo reprogramming. These studies have shown that open chromatin regions undergo extensive reprogramming during the development of mice from fertilized eggs into preimplantation embryos, and H3K4me3 and H3K27me3 histone modifications play an important role in the reprogramming process. The establishment of the $\mathrm{H} 3 \mathrm{~K} 4 \mathrm{me} 3$ modification is relatively rapid and tends to occur in promoter regions with higher $\mathrm{CpG}$ contents and lower DNA methylation levels, while the H3K27me3 modification is established slowly and tends to be established in promoter regions with a lower CpG content $[8,9]$.

Previous studies have shown that fetal oviduct epithelial cells (FOVs) exhibit a higher blastocyst formation rate than fetal fibroblasts (FFBs), but the individual birth rate obtained from the latter cell type is lower [10]. The reason for this difference is not clear. Compared with the study of a single type of epigenetic modification, studying the chromatin accessibility of the whole genome can elucidate the epigenetic abnormalities of cloned animals at a higher chromatin level. To this end, this study aimed to compare the differences in chromatin openness among different cloned bovine ear tissue samples derived from FOVs and FFBs via joint assay for transposase-accessible chromatin with high-throughput sequencing (ATAC-seq) and RNA-seq analyses and to analyze the expression of enzyme genes related to epigenetic modifications, such as the methylation and demethylation of histone $\mathrm{H} 3 \mathrm{~K} 4, \mathrm{H} 3 \mathrm{~K} 9$, and H3K27 as well as DNA methylation $(5 \mathrm{mC})$ and hydroxymethylation $(5 \mathrm{hmC})$. At the same time, $5 \mathrm{mC}$ and $5 \mathrm{hmC}$, a pair of closely related DNA methylation and demethylation modifications, were selected for further 
analysis. Our results will be useful for understanding the effects of epigenetic modification and the chromatin state of different donor cells in cloned animals and will provide a new theoretical basis for addressing bottlenecks imposed by issues such as abnormal development and low efficiency in somatic cloned animals.

\section{Materials And Methods}

\section{Establishment of cell lines and identification of mycoplasma}

One-month-old ear tissue from surviving cloned cattle was soaked in $70 \%$ alcohol for $30 \mathrm{~s}$. Several pieces of epidermal tissue were cut from the tissue with sterile scissors and placed in basic medium containing 10\% FBS (DMEM [CORNING, USA] + $1 \times$ double antibodies [New Cell Molecular Biotech Co., Ltd., China] + 1 $\times$ NEAA [Gibco, USA]). These tissues were thoroughly minced in $60 \mathrm{~mm}$ sterile culture dishes, placed in T25 culture flasks containing $5 \mathrm{ml}$ of $10 \%$ FBS (CORNING, USA) basic medium in batches, and cultured in a $37^{\circ} \mathrm{C} 5 \% \mathrm{CO}_{2}$ incubator (Thermo Fisher Scientific, USA). During this period, the medium was changed every two days. After the cells were completely confluent, they were digested and passaged with $0.25 \%$ trypsin (Gibco, USA). Cell cryopreservation was then used to resuspend digested cells in cryopreservation solution (70\% DMEM + 20\% FBS + 10\% DMSO [Sigma-Aldrich, USA]), and the cells were transferred to a liquid nitrogen tank for long-term preservation after programmed cooling.

Mycoplasma contamination affects not only cell activity but also subsequent ATAC-seq, so it was necessary to test for mycoplasma in cell line samples. Mycoplasma was identified by PCR with a primer targeting a 16S DNA sequence unique to mycoplasma. The cell culture medium was collected and centrifuged for $10 \mathrm{~min}$ at $\geq 10,000 \times \mathrm{g}$, only $50 \mu \mathrm{l}$ of the supernatant was retained, $2 \mu \mathrm{l}$ of which was employed as a template after boiling. After the addition of 2X PCR mix (Vazyme, China), the following program was performed in the PCR instrument (Eppendorf, Germany): $94^{\circ} \mathrm{C}$ for $30 \mathrm{~s}, 55^{\circ} \mathrm{C}$ for $30 \mathrm{~s}$, and $72^{\circ} \mathrm{C}$ for $30 \mathrm{~s}, 30$ cycles, which was followed by agarose gel electrophoresis. If mycoplasma existed treatment with mycoplasma remover was performed for at $10 \mu \mathrm{g} / \mathrm{ml}$ for 7-10 days, and the assay was repeated again until mycoplasma was removed. Then, a $1 \mu \mathrm{g} / \mathrm{ml}$ dose was used for maintenance culture.

\section{Establishment of ATAC-seq and data analysis}

Six cell lines of cloned cattle from different donor nuclei (three strains derived from fetal fibroblasts, numbered FFB1, FFB2, and FFB3, and another three strains derived from fetal oviduct epithelial cells, numbered FOV1, FOV2, and FOV3) were carefully digested with trypsin after mycoplasma assessment. After centrifugation at low temperature, the cells were resuspended in PBS for trypan blue (Thermo Fisher Scientific, USA) staining to determine cell activity. Cells with good cell activity were subjected to counting, and only approximately $5 \times 10^{4}$ cells were needed. After the cells were lysed with precooled lysis buffer, gently blown upon, and centrifuged, chromatin precipitates of good quality were obtained, and the samples were treated with Tn5 transposase to establish a transposition library. After PCR amplification 
and quality inspection, this library was sequenced using the Illumina HiSeq platform with $2 \times 150 \mathrm{bp}$ double-ended sequencing, and the data volume of each sample exceeded $20 \mathrm{G}$ bases. However, these data contained raw reads with linkers or low quality. To improve the quality for information analysis, it was necessary to use the software trim_galore

(http://www.bioinformatics.babraham.ac.uk/projects/trim_galore/) for quality filtering with the following parameters: $a$. remove sequencing primers; $b$. remove low-quality $(Q<10)$ sequences at the end of reads; and $c$. remove reads with fragments of $<35 \mathrm{bp}$ after $\mathrm{Ab}$ treatment). This finally resulted in $19.5 \mathrm{G}$ of clean bases per sample. MACS software (http://liulab.dfci.harvard.edu/MACS/) [28] was then used to identify rich regions of reads in a reference genome (i.e., peak calling) as a candidate open chromatin sites. Peak Annotator (v1.4) software [29] was used to annotate the genes upstream and downstream of the peaks, and the names and distances of adjacent genes on the positive and negative strands of the genome for each peak were then obtained. According to different donor nucleus sources, the samples could be classified into the FFB group (FFB1, FFB2, and FFB3 derived from fetal fibroblasts) or FOV group (FOV1, FOV2, and FOV3 derived from fetal oviduct epithelial cells) to conduct a differential peak analysis between samples.

\section{Establishment of RNA-seq and data analysis}

RNA was extracted from the above six cell lines using the TRIzol method, and the concentration and purity were determined using a NanoDrop 2000 microspectrophotometer (Thermo Fisher Scientific, USA). Then, $20 \mu \mathrm{g}$ of high-quality RNA was used to synthesize cDNA, which was subsequently sheared using an ultrasonic apparatus with the size controlled to 300-500 bp. After purification, amplification was carried out to complete library construction, and these libraries were then sequenced using an Illumina HiSeq system. The raw data obtained by sequencing needed to be filtered, and we removed the reads containing adapter sequences, single-ended sequencing reads with undetected bases (represented by $\mathrm{N}$ ) that exceeded the length of the reads by $10 \%$, and single-ended sequencing reads that contained low-quality bases that exceeded the length ratio of the reads by $50 \%$, finally producing clean reads. Then, a quality evaluation of the clean reads was conducted, including assessments of the production statistics of our sequencing data, the distribution of the sequence base content and quality, and the distribution of GC content in the sequencing data, to ensure that the obtained data were of high quality. The qualitycontrolled sequences were aligned to the reference genome sequence using HISAT2 and to the reference transcript sequence using Bowtie2 for subsequent differential expression analysis.

\section{Determining the content of $5 \mathrm{mc}$ and $5 \mathrm{hmc}$ in different samples}

Living and dead cloned bovine ear tissue samples were ground in liquid nitrogen, DNA was extracted using a DNA extraction kit (TIANGEN, China), and purity was determined by agarose gel electrophoresis. Then, the $5 \mathrm{mC}$ and $5 \mathrm{hmC}$ contents of the whole genome were determined in high-purity DNA. 5mC was detected by high-performance liquid chromatography. The DNA was hydrolyzed into single bases by using hydrofluoric acid, with $0.2 \%$ (volume fraction) phosphoric acid as the mobile phase. The hydrolysate was passed through an Ultimate Polar-RP chromatographic column $(4.6 \mathrm{~mm} \times 250.0 \mathrm{~mm}, 5$ 
$\mu \mathrm{m}$ ) at a flow rate of $1.2 \mathrm{ml} / \mathrm{min}$, and the detection wavelength was $273 \mathrm{~nm} . \mathrm{C}$ and $5 \mathrm{mC}$ standard solutions were prepared with concentrations of $0.5 \mathrm{mg} / \mathrm{L}, 1.0 \mathrm{mg} / \mathrm{L}, 2.0 \mathrm{mg} / \mathrm{L}, 5.0 \mathrm{mg} / \mathrm{L}, 10.0 \mathrm{mg} / \mathrm{L}, 20.0$ $\mathrm{mg} / \mathrm{L}$, and $50.0 \mathrm{mg} / \mathrm{L}$ to draw standard curves. To compare the results with the standard, the integrated area of $5 \mathrm{mC} /(5 \mathrm{mC}+\mathrm{C})$ was calculated to determine the overall methylation level of the genome. $5 \mathrm{hmC}$ was detected using a QTRAP 4000 LC/MS system from AB Sciex company. DNA was gradually enzymolyzed with nuclease S1, phosphatase, and snake venom phosphodiesterase, and the samples were recycled. The mixed mobile phase with a ratio of $2 \mathrm{mM}$ sodium acetate:methanol =2:10 was injected, and the total hydroxymethylation content was detected via the standard curve method.

\section{Results}

\section{The chromatin of surviving cattle cloned using FOVs as donor nuclei was more open than that of cattle derived from FFBs}

The insertion fragment size distribution in an ATAC-seq library represents the periodic change in the length of DNA (146 bp) wrapped around nucleosomes, and the proportion of fragments with increased number of nucleosome units was therefore decreased (Fig. 1A). Moreover, the majority of reads were distributed in the regions centered on the transcriptional start site (TSS) within 2000 bp of the site (Fig. 1B), which indicated that the ATAC-seq data were reliable. We also conducted an analysis of peaks showing differences between the FFB and FOV groups using the software package DiffBind, and the statistics of the peak numbers were significant (P-value $<0.05)$. We also found that 18,893 peaks were upregulated and that 21,136 peaks were downregulated in the FFB group relative to the FOV group (Fig. 1D), indicating that the chromatin openness of the FOV group was greater than that of the FFB group. We analyzed the regional distribution of peak areas in the genome using Annovar software as well as their proportions in each part of a gene. We found that the differential peaks were mainly concentrated in intergenic regions (67.94\%) and intronic regions (24.38\%), while the proportion of upstream and downstream regions was lower (Fig. 1E-F).

\section{The ATAC and gene expression analyses of cloned bovine ear tissues from homologous donor nuclei showed an obvious clustering tendency}

We next conducted an overall evaluation of the high-quality RNA-seq data obtained by filtering and found that the correlation between samples was high (Fig. 2A-B), indicating that the experimental results were reliable and that the selected samples were appropriate. To facilitate an analysis of differential expression levels between these two groups, we used RSEM to analyze the alignment results from Bowtie2 (http://bowtie-bio.sourceforge.net/bowtie2/index.shtml). We obtained the number of reads per sample aligned to each transcript, which was expressed as the value obtained fragments per kilobase per million bases (FPKM) conversion; the paired-end reads from the same fragment were counted as a single fragment, and the expression levels of genes and transcripts were obtained. We determined differential expression using the $\mathrm{R}$ package $\mathrm{DEseq} 2$, and the screening thresholds were an false discovery rate (FDR) $<0.05$ and a $\log _{2}$ fold-change $(F C)>1$ or $<-1$. The results showed that there were 1805 differentially 
expressed genes between the FFB and FOV groups, and there were 713 upregulated and 1092 downregulated differentially expressed genes in the FFB group relative to the FOV group (Fig. 2C). This indicated that gene expression activity was higher in the FOV group, which was consistent with our previous ATAC-seq results. In addition, based on the patterns in a cluster map of differentially expressed gene expression, we very intuitively observed significant clustering of gene expression between the two groups, although all of the data were derived from the same part of cloned cattle ear tissues with a "normal phenotype".

\section{Combined ATAC-seq and RNA-seq analysis of epigenetic modification-related enzyme genes}

To study the differences in the epigenetic modification of cloned cattle between the FFB and FOV groups, we analyzed many methylases and demethylases related to DNA methylation and histone modification, respectively. We selected the DNA methylase genes $D N M T 1, D N M T 3 A$, and DNMT3B, the DNA demethylase genes TET1, TET2, and TET3, the histone H3K4 methylase gene KMT2E, the H3K9 methylase geness SUV39H1, SUV39H2, and SETDB1, the H3K27 methylase gene EZH2, and the genes encoding the corresponding demethylating enzymes KDM5A, KDM4A, KDM3A, and KDM6A. Then, we analyzed the ATAC-seq and RNA-seq results for these genes (see Additional file 1, Table S1 and Table S2), selected the ATAC-seq peaks of some genes (Fig. 3A), and used the RNA-seq results of these enzymes (see Additional file 1, Table S3) to draw a heat map using TBTools (Fig. 3B). The ATAC-seq results showed that higher peaks generally appeared in the upstream and downstream regions of a gene (Fig. $3 \mathrm{~A}$, represented by the red box). The up- and downregulated regions of these epigenetic modification enzymes were largely concentrated in intron regions. The RNA-seq results (Fig. 3B, left) showed that the expression levels of genes related to promoting gene expression, such as $T E T 1,2,3, K D M 5 A$, and KDM6A, were relatively low, while those of $D N M T 1, E Z H 2$, and $K D M 4 A$ were higher. We standardized the expression of these genes and found that almost every gene exhibited a relatively higher expression in the FFB group than in the FOV group.

Based on the combined ATAC-seq and RNA-seq analysis, we found that the H3K27 methylase EZH2, which is related to gene expression inhibition, and the H3K9 methylase $\mathrm{SUV} 39 \mathrm{H} 2$, related to heterochromatin formation, were upregulated in the FFB group. These findings were consistent with the results showing that the chromatin of cloned animals in the FOV group was more open.

\section{$5 \mathrm{mc}$ and $5 \mathrm{hmc}$ levels in live and dead cattle cloned with different donor nuclei}

Next, we selected $5 \mathrm{mc}$ and $5 \mathrm{hmc}$, a pair of closely related DNA methylation and demethylation modifications, for further analysis. $5 \mathrm{hmc}$ is an important DNA demethylation intermediate. In organisms, $5 \mathrm{mc}$ is successively oxidized to $5 \mathrm{hmC}, 5$-formylcytosine $(5 \mathrm{fC})$, and 5-carboxylcytosine ( $5 \mathrm{caC}$ ) by the TET family proteins TET1, 2, and 3. The final step is conversion to common cytosine by base excision and repair (Fig. 4A) [12-15]. We first determined the $5 \mathrm{mC}$ and $5 \mathrm{hmC}$ contents of donor cell lines in ear tissues 
from six live cloned bovine individuals (FFB1, FFB2, FFB3, FOV1, FOV2, and FOV3) and five bovines produced via natural breeding (Fig. $4 \mathrm{~B}-\mathrm{C}$ ). The results showed that although the $5 \mathrm{mC}(\%)$ and $5 \mathrm{hmC}(\% \mathrm{o})$ contents of the genomes of all samples differed by an order of magnitude, which was consistent with the RNA-seq results showing that TET family protein expression was lower while the DNMT1 content was higher (Fig. 3B, left), there was a negative correlation of these samples overall. We also found that the content of $5 \mathrm{mC}$ in the FFB group was higher than that in the FOV group, and the same result was obtained in surviving individuals after overprogramming and differentiation (Fig. 4B), while the contents of $5 \mathrm{hmC}$ were almost equal (Fig. $4 \mathrm{C}$ ). Next, we examined the ear tissue samples of five dead FFB cloned cattle. The results showed that the $5 \mathrm{mC}$ contents of the ear tissue samples of living and dead cloned cattle were higher, but the $5 \mathrm{hmC}$ contents of dead cloned cattle whereas significantly lower than those of live cloned cattle. The $5 \mathrm{hmC}$ content of the surviving cloned cattle was higher than $4 \%$, and the $5 \mathrm{hmC}$ content of the dead cloned cattle was lower than 2\%o (Fig. 4D).

\section{Discussion}

To date, more than 20 species of mammals have been successfully cloned by somatic cell nuclear transfer [2], which plays an important role in many fields [3]. However, the birth rate of such animals is low [16], and the individuals that are born exhibit more health defects than naturally bred individuals, which seriously hinders their further development [17-19]. It is generally believed that this phenomenon is caused by aberrant epigenetic modification in the process of somatic reprogramming, and the abnormal epigenetic modification of reprogrammed cells also causes abnormalities in the forward programming process, which is the root cause of low birth rates and developmental defects in cloned animals.

Previous studies have focused on the effects of only a single type of epigenetic modification, but there are many kinds of epigenetic modifications, and they often coordinate with each other to make chromatin denser or looser by changing the structure of chromatin. Dense chromatin is correlated with gene silencing, while open chromatin promotes gene expression. Thus, compared with studying a single modification, the study of whole-genome chromatin openness may provide a more comprehensive understanding of the effects of epigenetic modification differences on cloned cattle. Therefore, in this experiment, ATAC-seq technology, which is currently a relatively advanced and efficient method for detecting chromatin openness [23], was used in a joint analysis with RNA-seq to provide a more comprehensive analysis of the differences in the epigenetic modifications of cloned cattle at the chromatin level. We found that cloned bovine samples from the same source showed a clustering tendency in both ATAC-seq and RNA-seq analyses, whereas the data of the cloned cattle from different sources were not clustered together. Even after the processes of reprogramming and differentiation, epigenetic imprinting was observed to affect each individual (i.e., the epigenetic memory phenomenon), similar to the findings of previous studies [24, 25].

We further analyzed the related genes encoding the enzymes responsible for epigenetic modifications. In this study, we analyzed 16 related genes, and our RNA-seq results showed that the expression levels of all 
genes were higher in the FFB group than in the FOV group. It was also found that the expression of enzymes related to inhibiting gene expression was higher than that of modifying enzymes related to promoting gene expression; e.g., the expression of TET1, 2, 3, KDM5A, KDM6A and other genes related to the promotion of gene expression was lower, while DNMT1, EZH2, and KDM4A exhibited higher expression. Since the expression level of inhibitory epigenetic modification-related enzymes in the FFB group was higher than that in the FOV group, we inferred that during the forward programming differentiation of somatic cloned cattle, with the continuous action of inhibitory epigenetic modificationrelated enzymes, some of the open chromatin in the genome would gradually become restricted. However, the expression of enzymes mediating inhibitory epigenetic modifications was higher in the FFB group than in the FOV group, so the chromatin was more open in cloned bovine ear tissue samples derived from the FOV group. In addition, our ATAC-seq results showed that although FOV chromatin was more open, the difference in chromatin openness between the cloned cattle from two different sources occurred in intergenic regions rather than in regions of gene expression. These data also showed that the large number of differences in intergenic regions did not directly affect the life or death of cloned cattle.

Studies have reported that regions with a high content of $5 \mathrm{hmC}$ modification are often accompanied by chromatin structure opening. Transcription factors and other cofactors can bind open DNA more easily, so genes near such regions tend to be highly expressed. We started with the analysis of the $5 \mathrm{hmc}$ modification of genomic DNA, which is an important demethylation modification that less studied than other modification in cloned animals, and we hoped to discover the role of $5 \mathrm{hmc}$ in somatic reprogramming and differentiation. By comparing the $5 \mathrm{mC}$ and $5 \mathrm{hmC}$ contents of the whole genomes of live and dead cloned cattle, we found that the $5 \mathrm{hmC}$ content of donor FOV cells was higher than that of FFB cells. The analysis of the individuals that survived after overprogramming and differentiation also showed that the $5 \mathrm{hmC}$ content of the FOV group was higher than that of the FFB group.

Our cloned bovine samples came from Dai Yunping's research group at the State Key Laboratory of Agrobiotechnology, China Agricultural University. As early as 2004, Professor Dai Yunping was studying the effects of different donor cell types on the production efficiency of somatic cloned cattle. By referring to their research [10] in combination with the results of our study, we found that FOV cells presented a significant advantage in terms of the blastocyst development rate, but the final individual birth rates were not as high as those obtained from FFB cells. Combined with the transcriptome sequencing results of this study, it could be inferred that aberrant modification during reprogramming influenced forward programming differentiation. We know that normal zygotic development proceeds through two stages: reprogramming and forward programming differentiation [3]. The early stage of SCNT requires reprogramming, and all previous epigenetic markers need to be removed from the cells need before they can develop normally to the blastocyst stage. At this stage, the FOV group exhibited a more open chromatin state (its $5 \mathrm{hmC}$ content was higher than that of the FFB group, suggesting that the chromatin was more open), which may have promoted this process, and showed a higher blastocyst formation rate. However, in the subsequent stage of differentiation and development, this relatively open chromatin may impede the process of differentiation, and the expression levels of enzymes mediating epigenetic modifications related to gene expression inhibition were also lower in the FOV group. Therefore, some 
genes that need to be silenced may not be shut down in time or may maintain high expression levels, ultimately leading to lower individual survival rates and developmental abnormalities, which may be similar to the results of previous H3K4me3 studies [5].

In conclusion, we hypothesized that differences in epigenetic modifications existed in cloned cattle because of their different donor cell types, and even after reprogramming and differentiation, some of these differences were retained in the cloned animals. In the reprogramming process of somatic cloned cattle, a more open chromatin state was beneficial for reprogramming. As a result, we further hypothesized that the high expression of epigenetic modification-related enzymes that promote gene expression or low expression of enzymes mediating inhibition-related modifications would facilitate the preimplantation development of somatic cloned cattle. In the process of differentiation and development, some open chromatin needs to be closed via a specific process, which occurs via the gradual addition of inhibitory epigenetic modifications that antagonize chromatin opening. When a chromatin region that needs to be closed cannot be closed in time, it may cause the abnormal development or death of cloned animals. Thus, in the process of somatic reprogramming, greater chromatin openness can promote the formation of blastocysts. However, in the development and differentiation period, this relatively open chromatin impedes the normal development of cloned animals.

\section{List Of Abbreviations}

SCNT: Somatic cell nuclear transfer; FFB: Fetal fibroblasts; FOV: Fetal oviduct epithelial cells; ATAC-seq: Assay for Transposase-Accessible Chromatin with high throughput sequencing; 5hmC: 5hydroxymethylcytidine; 5mC: 5-methylcytosine; TSA: Trichostatin A; TSS: Transcriptional start site; FPKM: Fragments per kilobase per million bases; FDR: False discovery rate; FC: fold-change; $5 f C$ : 5 -

formylcytosine; 5caC: 5-carboxylcytosine; TES: Transcription end site; DEG: Differentially expressed gene; C-FFB/FOV = Control-FFB/FOV; NT-FFB/FOV = Nuclear transfer-FFB/FOV.

\section{Declarations}

\section{Ethics approval and consent to participate}

All operations in this research strictly abide by the ordinances of the Administration of Laboratory Animals (Ministry of Science and Technology, China, revised 2004). All animal experimental procedures were authorized by the Committee on the Ethics of Animal Experiments of Laboratory Animals of the Henan University.

\section{Consent for publication}

Not applicable.

\section{Availability of data and material}


The datasets used and/or analyzed during the current study are available from the corresponding authors upon reasonable request.

\section{Competing interests}

Not applicable.

\section{Funding}

This work was supported by National Natural Science Foundation of China (31771647) and key Scientific and Technological Special Projects of Henan Province(201300111200).

\section{Authors' contributions}

HQM and TH prepared and analysed the ATAC-seq and RNA-seq materials. JZ determined the 5hmC content in the genome. JHL determined the $5 \mathrm{mC}$ content in the genome. CYY and SHC took samples and cultured cell. CL took charge of the nutrition and reproduction of cattle. GSC and YYB designed experiment, wrote paper, and disscussed.

\section{Acknowledgements}

This work was supported by the National Natural Science Foundation of China (31771647) and Key Scientific and Technological Special Projects of Henan Province(201300111200).

\section{References}

1. Kang YK, K.D., Park JS, Choi YH, Chung AS, Lee KK, Han YM (2001) < Aberrant methylation of donor genome in cloned bovine embryos..pdf>.

2. Rodriguez-Osorio N, et al. Reprogramming mammalian somatic cells. Theriogenology. 2012;78(9):1869-86.

3. Yang $X$, et al. Nuclear reprogramming of cloned embryos and its implications for therapeutic cloning. Nat Genet. 2007;39(3):295-302.

4. Matoba S, et al. Embryonic development following somatic cell nuclear transfer impeded by persisting histone methylation. Cell. 2014;159(4):884-95.

5. Hormanseder E, et al. (2017) H3K4 Methylation-Dependent Memory of Somatic Cell Identity Inhibits Reprogramming and Development of Nuclear Transfer Embryos. Cell Stem Cell 21 (1), 135-43 e6.

6. Zhang M, et al. Defective Chromatin Structure in Somatic Cell Cloned Mouse Embryos. J Biol Chem. 2009;284(37):24981-7.

7. Zhou C, et al. H3K27me3 is an epigenetic barrier while KDM6A overexpression improves nuclear reprogramming efficiency. Faseb j. 2019;33(3):4638-52.

8. Liu X, et al. Distinct features of H3K4me3 and H3K27me3 chromatin domains in pre-implantation embryos. Nature. 2016;537(7621):558-62. 
9. Wu J, et al. The landscape of accessible chromatin in mammalian preimplantation embryos. Nature. 2016;534(7609):652-7.

10. Gong G, et al. Generation of cloned calves from different types of somatic cells. Sci China C Life Sci. 2004;47(5):470-6.

11. Langmead B, Salzberg SL. Fast gapped-read alignment with Bowtie 2. Nat Methods. 2012;9(4):3579.

12. Globisch D, et al. Tissue distribution of 5-hydroxymethylcytosine and search for active demethylation intermediates. PLoS One. 2010;5(12):e15367.

13. Ito $S$, et al. Tet proteins can convert 5-methylcytosine to 5-formylcytosine and 5-carboxylcytosine. Science. 2011;333(6047):1300-3.

14. He YF, et al. Tet-mediated formation of 5-carboxylcytosine and its excision by TDG in mammalian DNA. Science. 2011;333(6047):1303-7.

15. Nabel CS, Kohli RM. Molecular biology. Demystifying DNA demethylation. Science. 2011;333(6047):1229-30.

16. Rodriguez-Osorio N, et al. Reprogramming mammalian somatic cells. Theriogenology. 2012;78(9):1869-86.

17. Young LE, et al. Large offspring syndrome in cattle and sheep. Rev Reprod. 1998;3(3):155-63.

18. Farin PW, et al. Errors in development of fetuses and placentas from in vitro-produced bovine embryos. Theriogenology. 2006;65(1):178-91.

19. Su JM, et al. Expression and methylation status of imprinted genes in placentas of deceased and live cloned transgenic calves. Theriogenology. 2011;75(7):1346-59.

20. Chammas $\mathrm{P}$, et al. Engaging chromatin: PRC2 structure meets function. Br J Cancer. 2020;122(3):315-28.

21. Weinberg DN, et al. The histone mark H3K36me2 recruits DNMT3A and shapes the intergenic DNA methylation landscape. Nature. 2019;573(7773):281-6.

22. Shahid Z, et al. Genetics, Histone Code. In: StatPearls, StatPearls Publishing Copyright @ 2020. StatPearls Publishing LLC; 2020.

23. Buenrostro JD, et al. (2015) ATAC-seq: A Method for Assaying Chromatin Accessibility Genome-Wide. Curr Protoc Mol Biol 109, 21.29.1-21.29.9.

24. Ng RK, Gurdon JB. Epigenetic inheritance of cell differentiation status. Cell Cycle. 2008;7(9):1173-7.

25. Ng RK, Gurdon JB. Epigenetic memory of active gene transcription is inherited through somatic cell nuclear transfer. Proc Natl Acad Sci U S A. 2005;102(6):1957-62.

26. Neri F, et al. Genome-wide analysis identifies a functional association of Tet1 and Polycomb repressive complex 2 in mouse embryonic stem cells. Genome Biol. 2013;14(8):R91.

27. Hahn MA, et al. Dynamics of 5-hydroxymethylcytosine and chromatin marks in Mammalian neurogenesis. Cell Rep. 2013;3(2):291-300.

28. Zhang Y, et al. Model-based analysis of ChIP-Seq (MACS). Genome Biol. 2008;9(9):R137. 
29. Salmon-Divon M, et al. PeakAnalyzer: genome-wide annotation of chromatin binding and modification loci. BMC Bioinformatics. 2010;11:415.

\section{Figures}

A

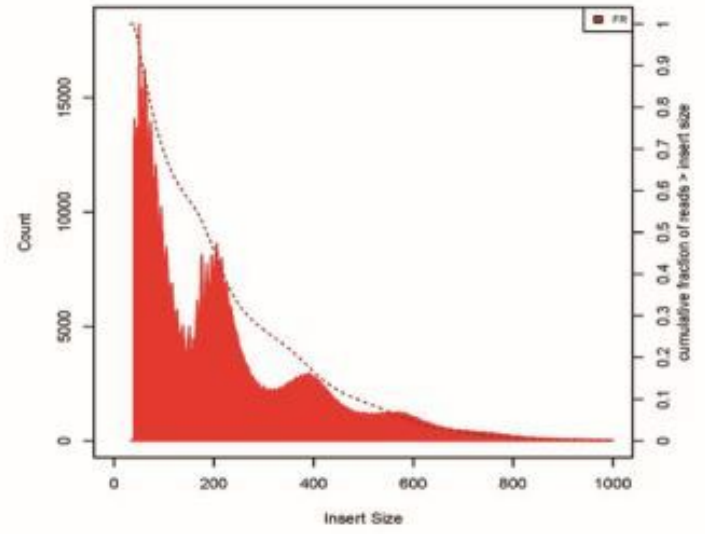

B
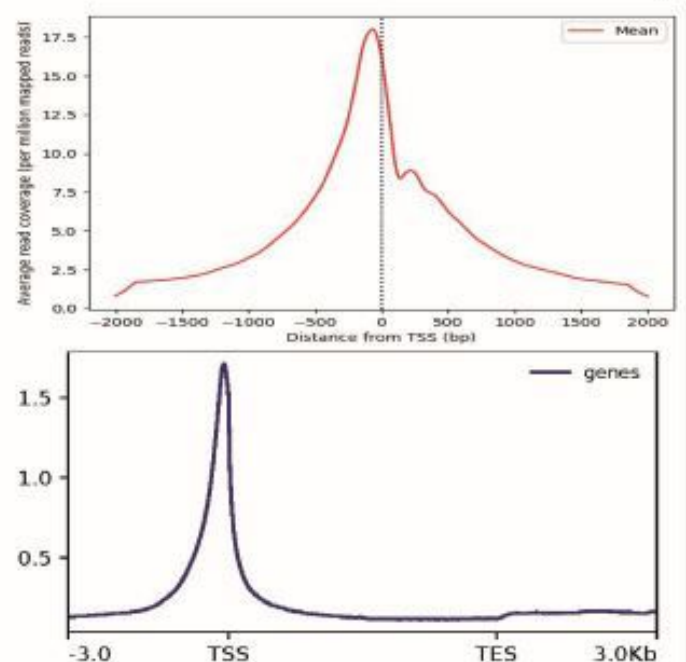

E

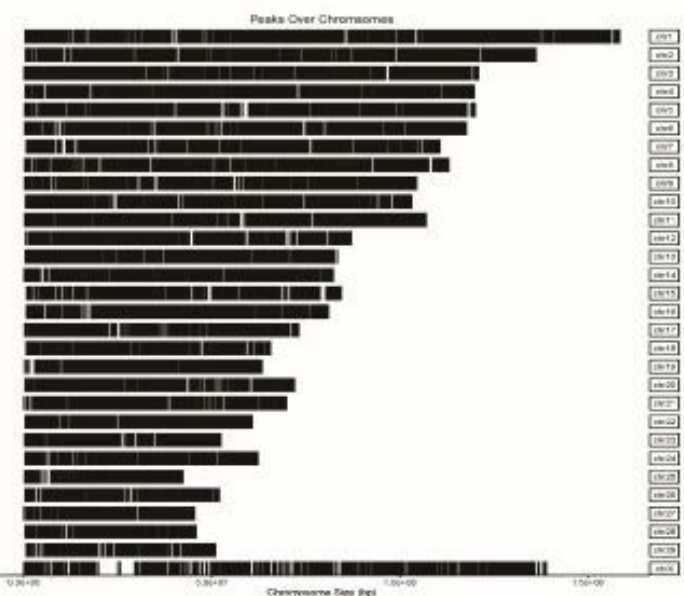

C

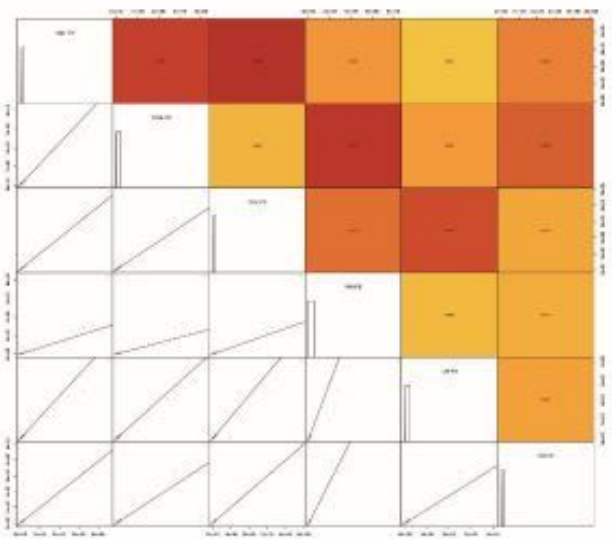

D
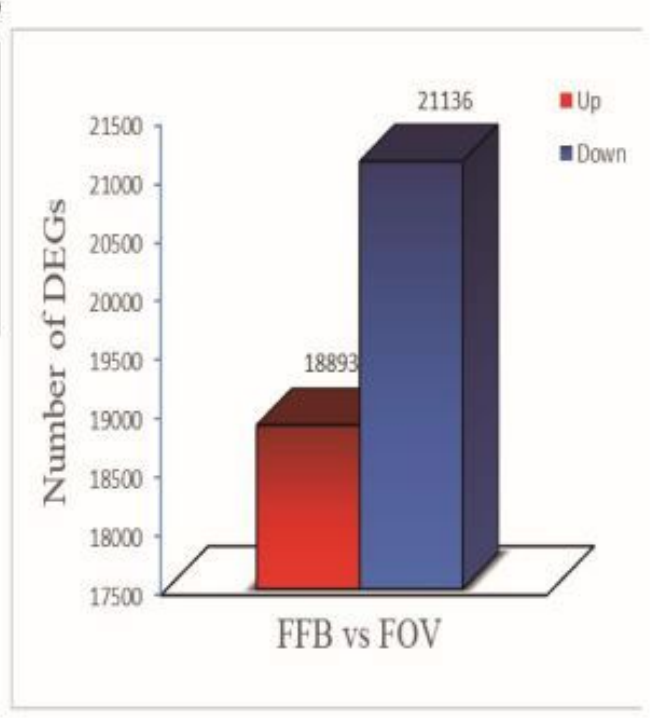

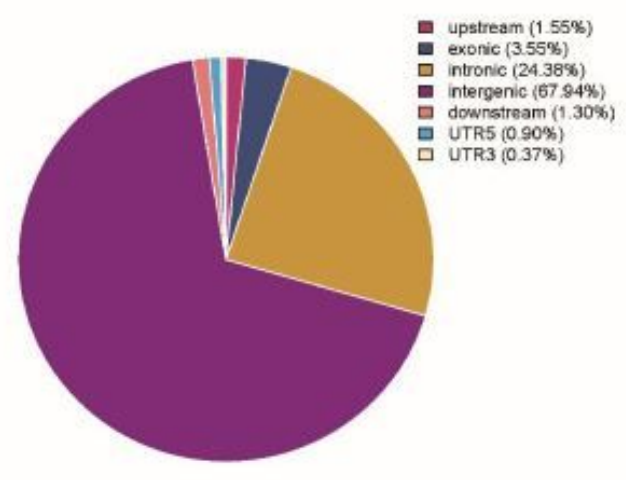

Figure 1 
Analysis of ATAC-seq results: A) Insertion fragment size distribution in the ATAC-seq library represents a periodic change in the length of DNA (146 bp) wrapped around nucleosomes, and the proportion of fragments with more nucleosome units decreased in turn; $\mathrm{B}$ ) the signal distribution of reads at the transcription start site (UP) and the genome (down), the signal distributed in the region 2000 bp above and below the center of the transcription start site (TSS), and the genome distributed near the TSS and the transcription end site (TES); C) Correlation scatter diagram between samples of the FFB and FOV groups; the closer the correlation coefficient is to 1 , the higher the similarity of the expression patterns between samples; D) Comparison of differential genes between the FFB and FOV groups; E, F) Distribution of differential genes between the FFB and FOV groups. E shows the distribution of differential peaks on 30 pairs of bovine chromosomes. The white stripe represents the position of differential peaks on chromosomes. $F$ shows the distribution of differential peaks in different regions of the genome (including upstream regulatory regions, introns, exons, intergenic regions, downstream regulatory regions, 5'untranslated regions, and 3'untranslated regions). 
A

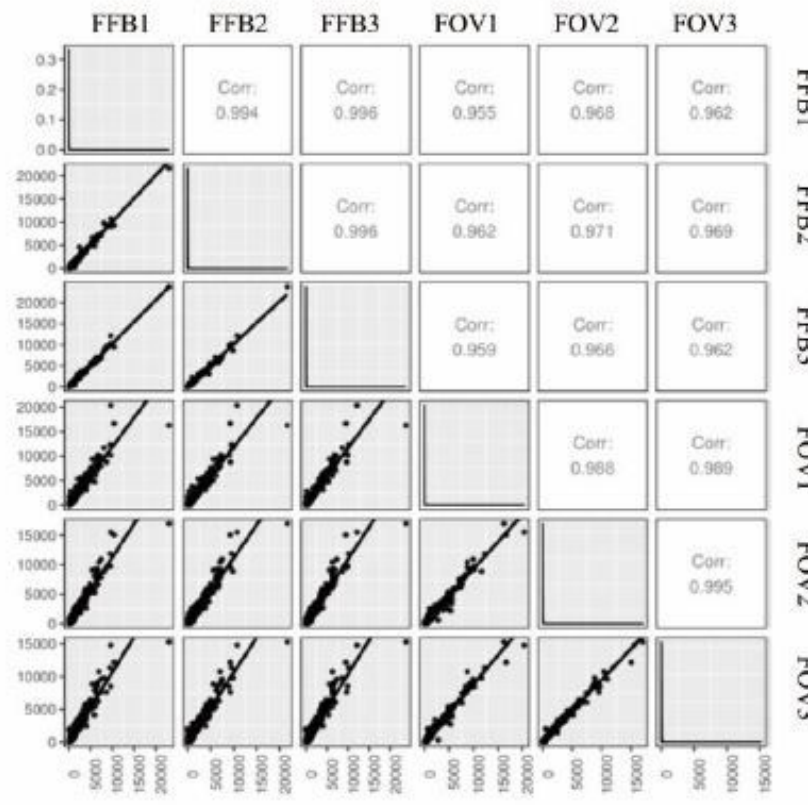

C

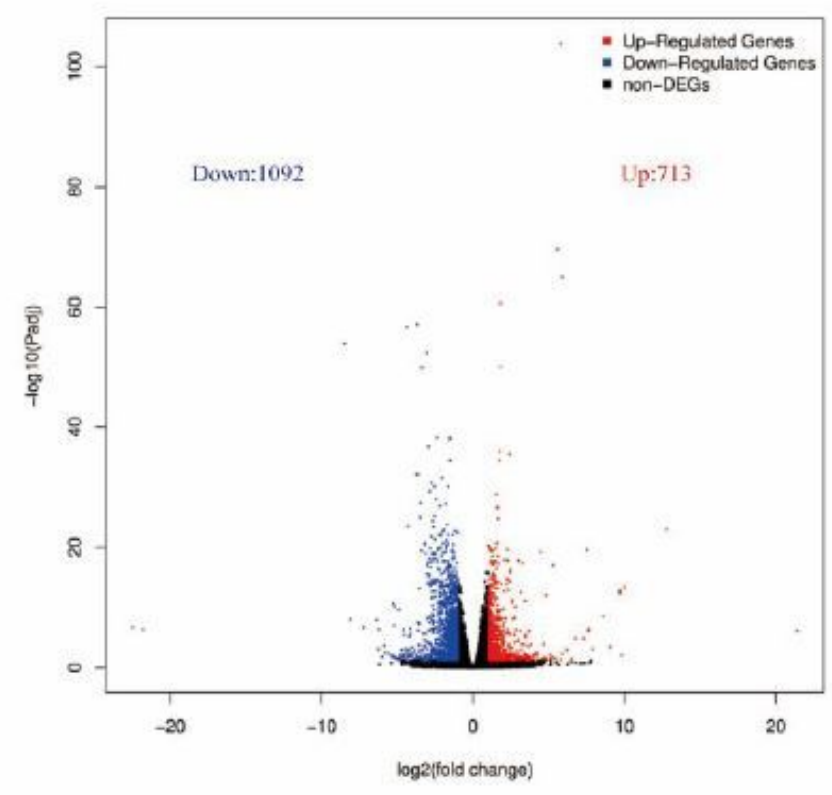

B

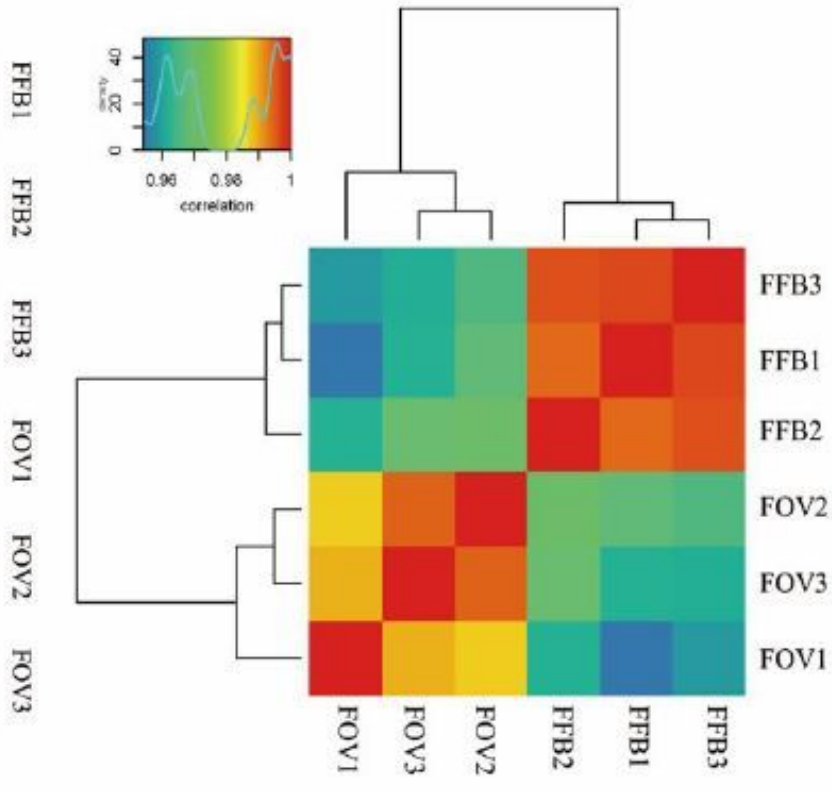

D

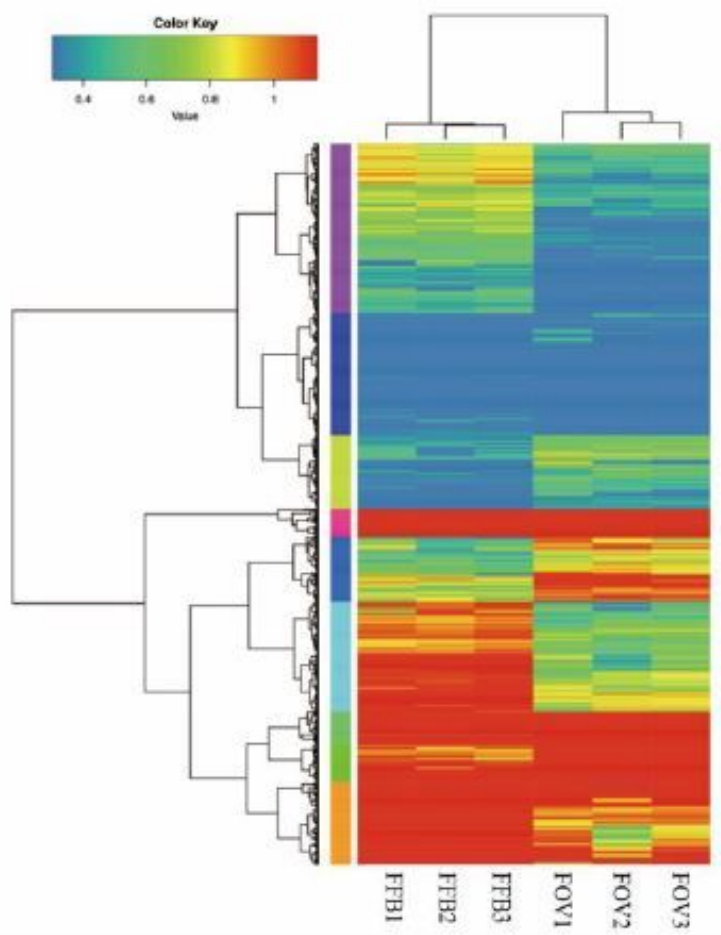

\section{Figure 2}

Quality control and analysis of RNA-seq results; A) Correlation scatter plot between samples of the FFB and FOV groups; B) Sample correlation heat map; C) Volcano plot of differentially expressed genes (DEGs) distribution in FFB vs. FOV; 713 genes were up-regulated (red) and 1092 were down-regulated

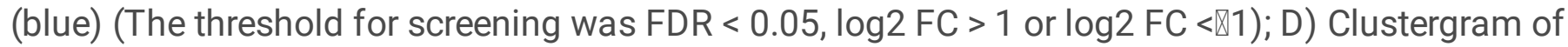


differential gene expression patterns. On the left, the differential genes are divided into eight subclusters according to their clustering situation and are represented by different colors.

A
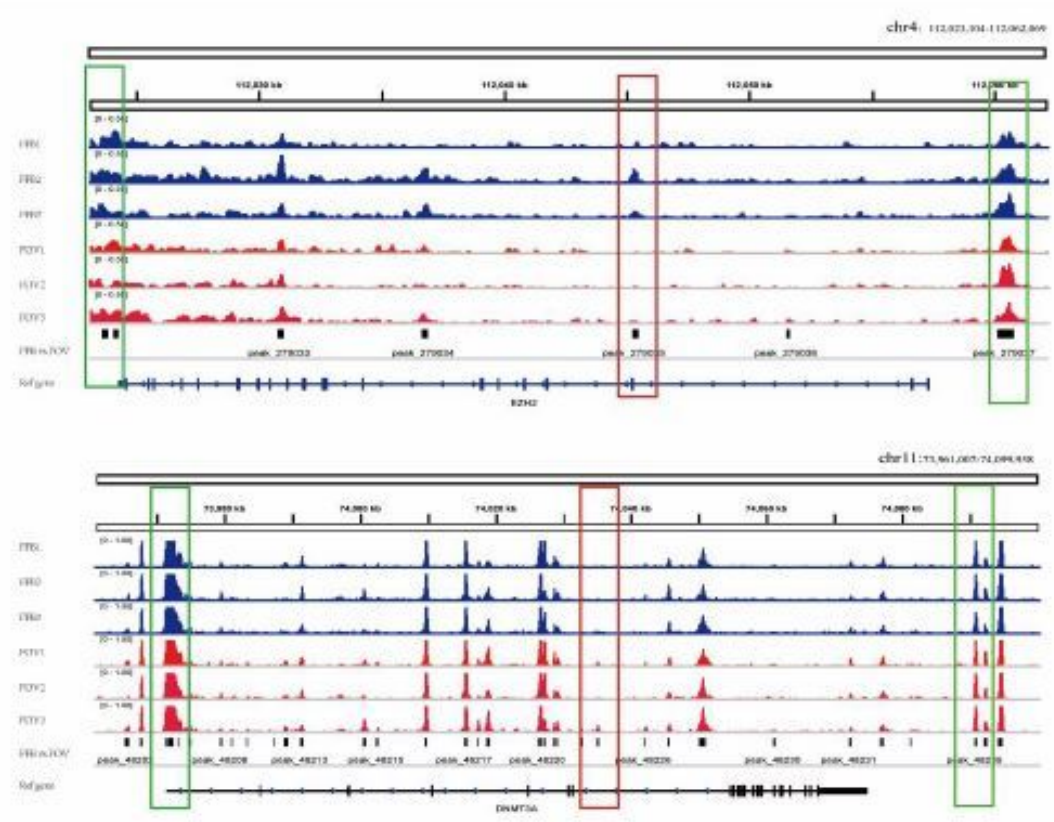

B

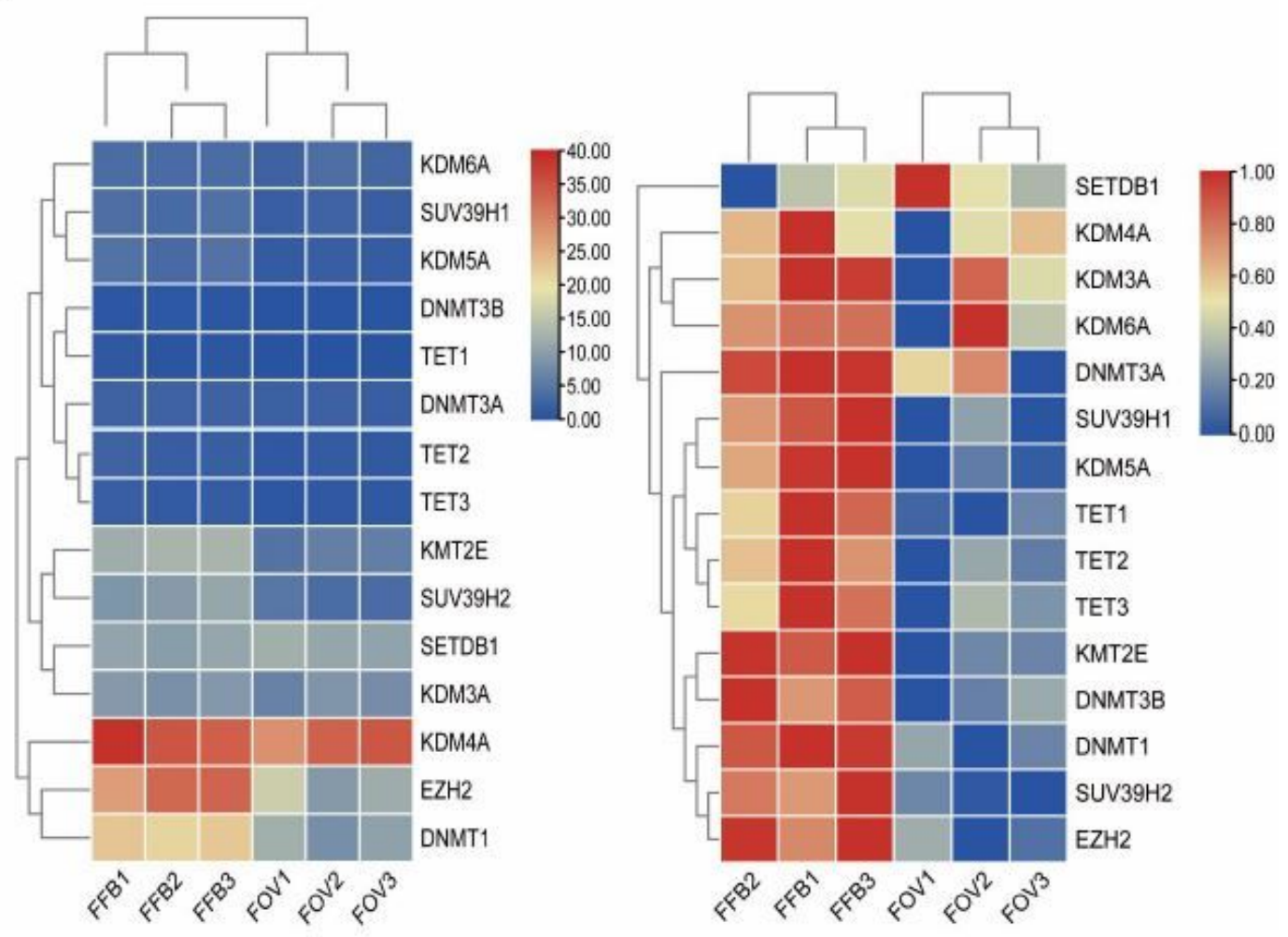

Figure 3

Joint analysis of ATAC-seq and RNA-seq: A) Differential peak distribution map of ATAC-seq between EZH2 and DNMT3A (up: EZH2, down: DNMT3A, the red box indicates up- or down-regulated peak positions, and the green box indicates the peak conditions in the upstream and downstream of a gene); 
B) Heat map of the expression of epigenetic modified enzyme-related genes; the left shows the original expression of each gene, and the right shows the gene expression after standardizing the original data.

A

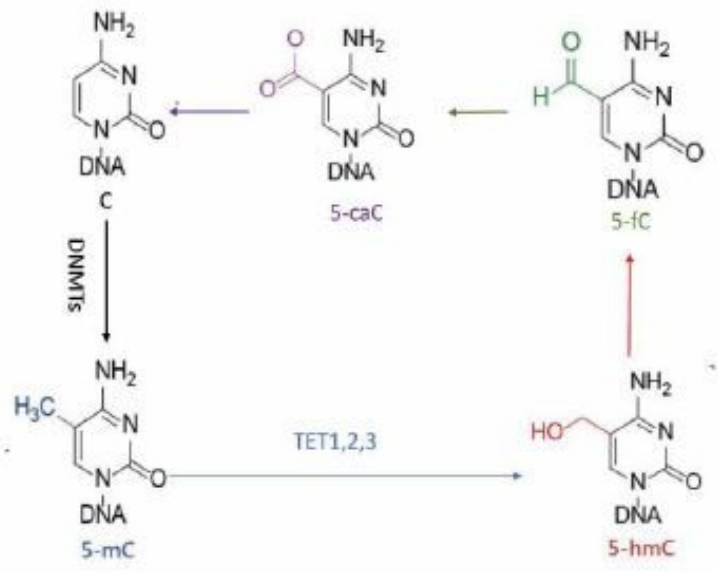

$\mathrm{C}$

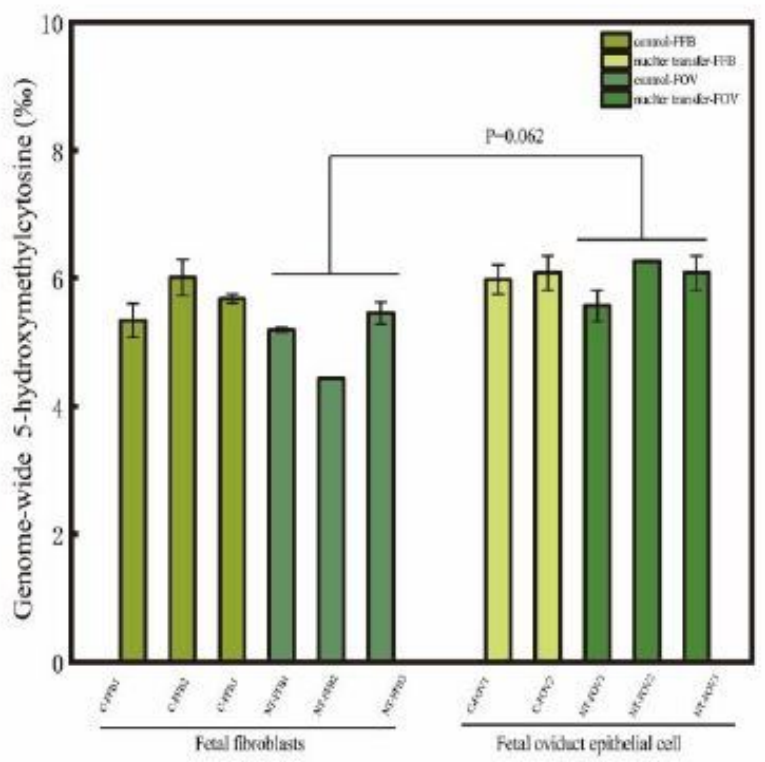

B

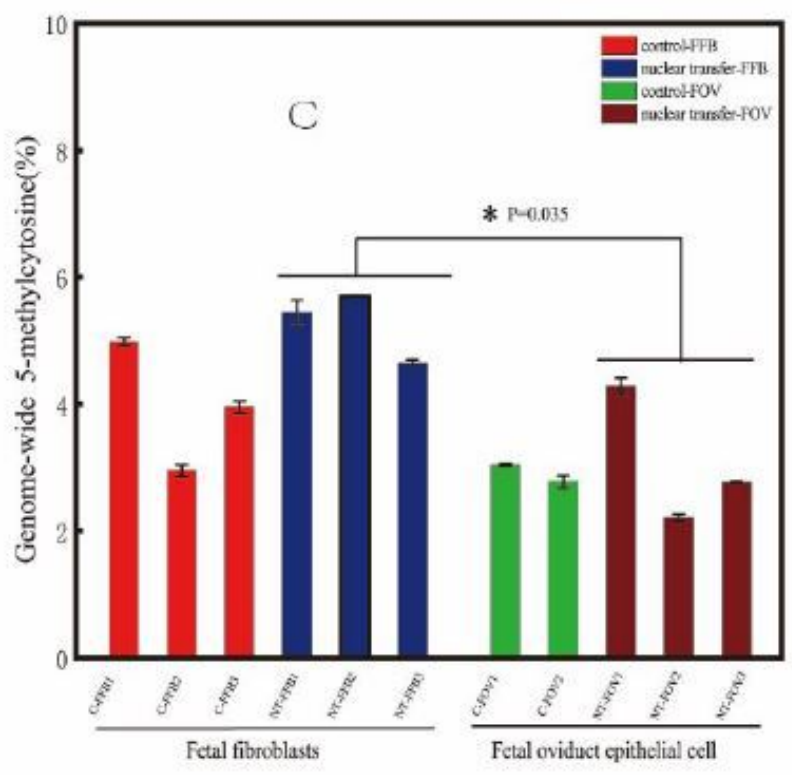

$\mathrm{D}$

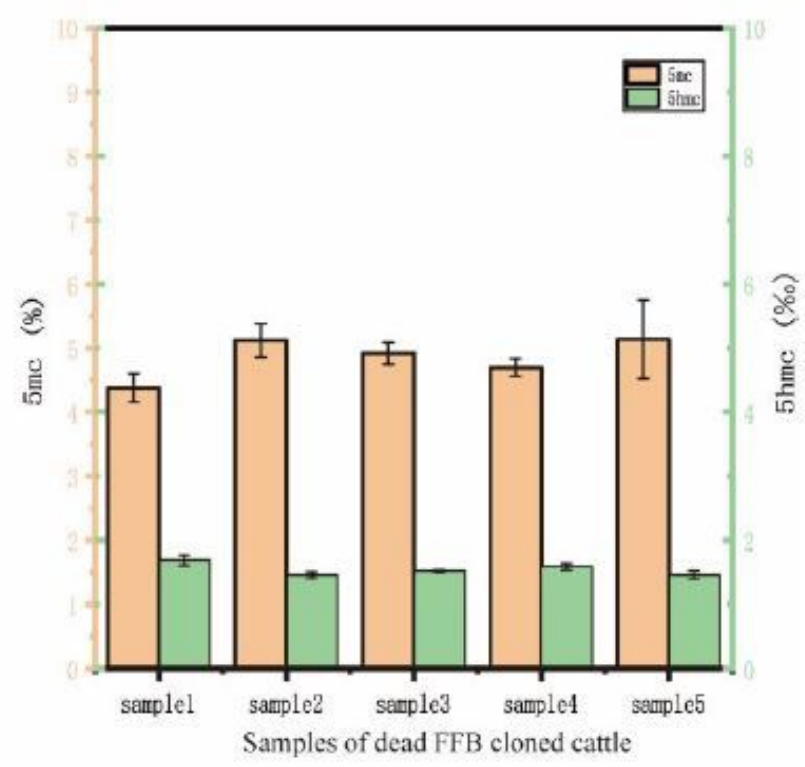

Figure 4

Analysis of the $5 \mathrm{mC}$ and $5 \mathrm{hmC}$ content in cloned bovine samples: A) $5 \mathrm{mC}$ demethylation process dependent on TET 1, 2, 3 proteins (C: cytosine, 5hmC: 5-hydroxymethylcytosine, 5fC: 5-formylcytosine, 5caC: 5-carboxylcytosine, 5mC: 5 methylcytosine) B) $5 \mathrm{mC}$ content in cloned bovine donor cell lines and 
surviving cloned bovine ear tissue samples (FFB: Fetal fibroblasts, FOV: Fetal oviduct epithelial cells, CFFB/FOV = control-FFB/FOV: Natural breeding bovine donor cell line, NT-FFB/FOV = nuclear transferFFB/FOV: Ear tissue samples of surviving individuals after nuclear transplantation); C) $5 \mathrm{hmC}$ content in cloned bovine donor cell lines and surviving cloned bovine ear tissue samples (FFB: Fetal fibroblasts, FOV: Fetal oviduct epithelial cells, C-FFB/FOV = control-FFB/FOV: Natural breeding bovine donor cell line, NT-FFB/FOV = nuclear transfer-FFB/FOV: Ear tissue samples of surviving individuals after nuclear transplantation); D) Determination of $5 \mathrm{mc}$ and $5 \mathrm{hmc}$ in dead cloned bovine ear tissue samples with FFB as donor cells. (The contents of $5 \mathrm{mC}$ and $5 \mathrm{hmC}$ are expressed by yellow and green, and the coordinate axes are also expressed by the corresponding colors, and their units are \% and \%o, respectively.)

\section{Supplementary Files}

This is a list of supplementary files associated with this preprint. Click to download.

- Additionalfile1.pdf 